the pyrex filter is removed to allow color center formation, a train of $Q$-switch pulses is obtained. The glass must be exposed to the short wavelength u.v. from the lamp to produce $Q$-switching. If glass which has previously been darkened is placed inside a pyrex tube normal pulses result. This establishes that additional short lived color centers are required to produce self $Q$-switched pulses.

In another experimental setup a rod $60 \mathrm{~cm}$ long had its central length of $45 \mathrm{~cm}$ pumped by a $6 \mathrm{~mm}$ diameter xenon flashlamp. The rod diameter was $6 \mathrm{~mm}$, and the rod and flashtube were close wrapped with silver foil under water. The power supply consisted of $760 \mu \mathrm{F}$ in series with $120 \mu \mathrm{H}$ charged to $3.8 \mathrm{kV}$. The rod had a full reflector at one end and at the exit end only the $4 \%$ Fresnel reflection due to the glass-air interface. Fig. 1B and $1 \mathrm{C}$ show, respectively, the time traces produced after 40 flashes and 60 flashes. After 86 shots, only $3 Q$-switched pulses were produced with a separation between pulses of $180 \mu \mathrm{sec}$. The three pulses in fig. $1 \mathrm{D}$ are superimposed because the time between pulses is longer than the sweep time and the oscilloscope was triggered by each of the individual pulses. The full width at half maximum of the first and n. rrowest pulse is 35 nsec. After 103 flashes only one pulse was produced. The decrease in the number of pulses on repeated flashing is due to the accumulation of the stable, visible color centers, which lower the pumping efficiency. For some of the more intense pulses the rise time was slightly shorter than the fall time. This is the case when the gain required to overcome the saturable loss is comparable to the constant losses of the cavity [8].

The authors gratefully acknowledge R.Graf and G. Granitsas for making the glass, N. Guertin for technical assistance, and G. Young for helpful discussions.

\section{References}

1. R.W. Hellwarth, Advances in quantum electronics, Ed. F.J.Singer (Columbia University Press, New York, 1964) p. 334.

2. G. Bret and F. Gires, Appl. Phys. Letters 4 (1964) 175.

3. L.G. Cross and L.A.Cross, "A Q-switched ruby oscillator using a saturable filter" paper presented at IEEE, Cincinnati, Ohio, April 7, 1964.

4. B.H.Soffer and R.H.Hoskins, Nature 204 (1964) 276.

5. N.T. Melamed and C.Hirayama, Appl.Phys. Letters 6 (1965) 43.

6. R. Yokota, Phys. Rev. 95 (1954) 1145.

7. A. Kats and J.M.Stevels, Philips Research Repts. 11 (1956) 115 .

8. A.Szabo and R.A.Stein, J.Appl.Phys. 36 (1965) 1562 .

\title{
X-RAY MICROSCOPY BY SUCCESSIVE FOURIER TRANSFORMATION II. AN OPTICAL ANALOGUE EXPERIMENT *
}

\author{
J.T.WINTHROP and C.R. WORTHINGTON \\ Department of Physics, University of Michigan, \\ Ann Arbor, Michigan 48104
}

Received 3 May 1966

An optical analogue experiment related to a method of X-ray microscopy by successive Fourier transformation is described. Some experimental design features are discussed.

The operation of a two-step diffraction microscope $[1,2]$ using $X$-rays and light has often been considered. A major difficulty is the resolution

\footnotetext{
* This work was aided by U.S. Public Health Service Grant GM 09796.
}

attainable with existing $\mathrm{X}$-ray sources and film. In an attempt to circumvent this difficulty we have proposed two methods of X-ray microscopy by successive Fourier transformation [3]. In this letter we describe an optical analogue experiment that relates to one of these methods, the pinhole 


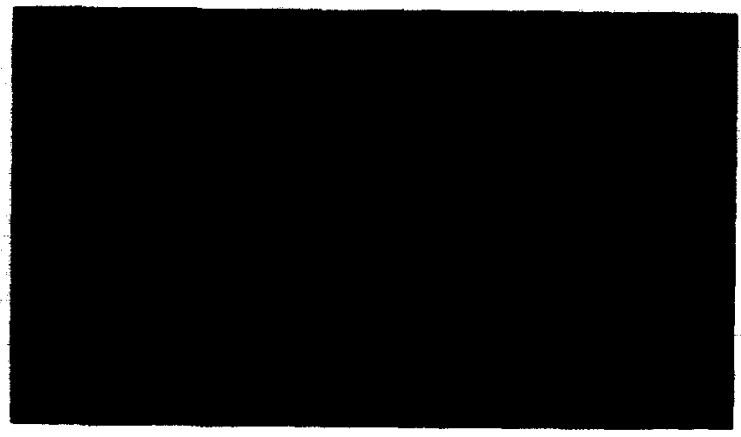

Fig. 1. Photographic enlargement of mask containing a triangular array of pinholes and a reference pinhole of diameter $\delta \approx \frac{1}{2} \mathrm{~mm} ; b \approx 14 \mathrm{~mm}$ is the distance from the pinhole to the far edge of the triangular array.

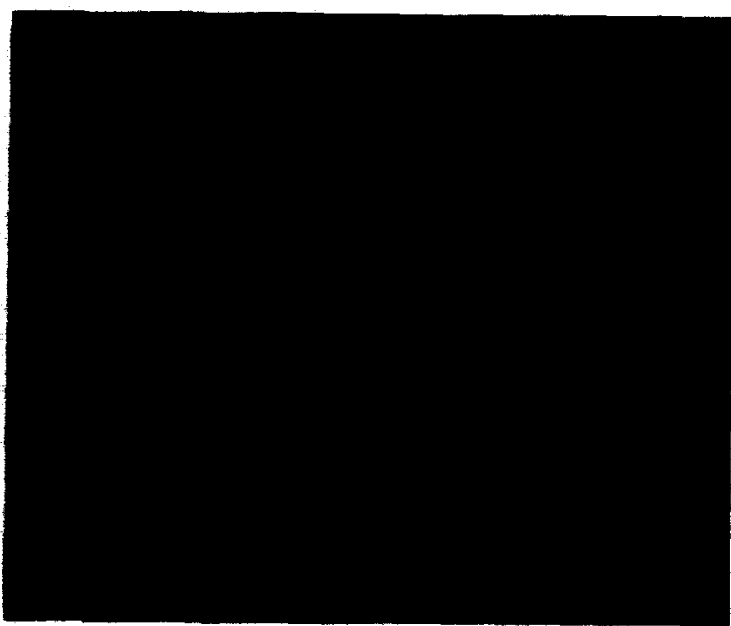

Fig. 2. The diffraction pattern of the mask obtained using the optical diffractometer. This is a Fouriertransform hologram.

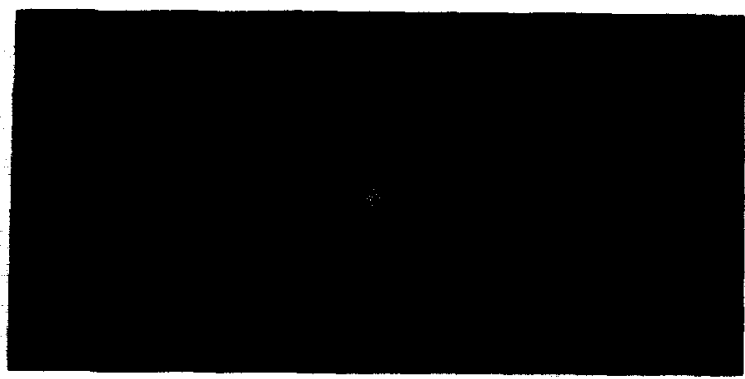

Fig. 3. The diffraction pattern of the hologram obtained using the optical diffractometer. This is the optical reconstruction.

method. We also present some further considerations on the design of the experimental setup and on the theoretical resolution.
The arrangement of components in the pinhole method comprises a Fourier-transform X-ray diffraction microscope $[3,4]$. The opaque object plane contains a small pinhole of diameter $\delta$ and an aperture of diameter $A$ over which the object is placed. The aperture and pinhole are separated, center-to-center, by the vector distance a. A Fourier-transform hologram of the object is obtained by recording the far-field $\mathrm{X}$-ray diffraction pattern of the object and reference pinhole. The far-field condition is

$$
1 / \lambda \mid 1 / p+1 / q ! b^{2} \leqslant 1,
$$

where $\lambda$ is the $X$-ray wavelength, $p$ is the distance from the source to the object plane, $q$ is the separation of the object and film planes, and $b$ is the distance from the pinhole to the far edge of the aperture. The amplitude transmittance of the hologram, $t_{\mathrm{H}}$, has the form

$t_{\mathrm{H}}=\left|T_{\mathrm{o}}\right|^{2}+\left|T_{1}\right|^{2}+2 \operatorname{Re}\left[T_{1}^{*} T_{\mathrm{o}} \exp \left(-\mathrm{i} 2 \pi \boldsymbol{a} \cdot \boldsymbol{r}^{*}\right)\right]$, where $\boldsymbol{r}^{*}=\boldsymbol{r} / \lambda q$, and where $T_{\mathrm{o}}\left(\boldsymbol{r}^{*}\right) \exp \left(-12 \pi \boldsymbol{a} \cdot \boldsymbol{r}^{*}\right)$ and $T_{1}\left(r^{*}\right)$ are the Fourier transforms of the pinhole transmittance function $t_{\mathrm{O}}(\boldsymbol{r}-\boldsymbol{a})$ and the object transmittance function $t_{1}(r)+$. Reconstruction by optical Fourier transformation gives the amplitude distribution

$$
T_{\mathrm{H}}=A_{\mathrm{O}}(\boldsymbol{u})+A_{1}(\boldsymbol{u})+R(\boldsymbol{u}-\boldsymbol{a})+R^{*}(-\boldsymbol{u}-\mathbf{a}),
$$

where $\boldsymbol{u}=\boldsymbol{r} / M$ and $M$ is the overall magnification [3]. The first two terms are the pinhole and object autocorrelation functions; these functions are contained within a circular region of diameter $2 M A$ centered at the origin. The two remaining terms describe magnified images of amplitude $R(\boldsymbol{u})=t_{\mathrm{o}}(\boldsymbol{u}) * t_{1}^{*}(-\boldsymbol{u})$ and $R^{*}(-\boldsymbol{u})=t_{\mathrm{o}}(-\boldsymbol{u}) * t_{1}(\boldsymbol{u})$, which are displaced from the origin by vector distances $M a$ and $-M a$ respectively. Separation of the reconstruction images from the autocorrelation pattern is obtained if $b>2 A$.

The optical analogue experiment is performed as follows. An opaque mask containing a triangular array of pinholes and a reference pinhole is placed in an optical diffractometer (R. B. Pullin and Co., London), which has a yellow-filtered

† The recording of $t_{\mathrm{H}}$ implies the following restrictions on film resolving power $N$, angular source size $d / p$, and $\Delta \lambda / \lambda$ :

$$
\begin{aligned}
N & \geqslant b / \lambda q, \\
d / p & \leqslant \lambda / 2 b, \\
\Delta \lambda / \lambda & \leqslant \delta / b .
\end{aligned}
$$

Hence by suitable choice of $b$ the recording of the fine details in $t_{\mathrm{H}}$ is possible with real X-ray sources and film. 
mercury-arc source. The diffractometer records the far-field diffraction pattern of the mask; this record when reproduced as a positive transparency is the Fourier-transform hologram + . An optical reconstruction is produced in the diffractometer by replacing the object mask with the hologram and recording the far-field diffraction pattern. A photograph of the mask is shown in fig. 1. The Fourier-transform hologram is shown in fig. 2. The Young's interference fringes originating from the separation of the object and reference pinhole are clearly seen. The optical reconstruction is shown in fig. 3. The autocorrelation of the triangular array covers the central region; this pattern is analogous to the Patterson diagram of crystallography. The separated reconstructions of the triangular array are well displaced from the autocorrelation pattern and have a center of symmetry with respect to the origin.

We note that optical demonstrations of Fouriertransform holography have also been given by Leith and Upatnieks [5] and by Vander Lugt [6]. The present demonstration differs in that a nonlaser source is used and the reference wave is produced by diffraction at a pinhole in the object plane. Hence there is correspondence to the X-ray case.

The diffractometer is a convenient device for recording optical Fourier-transform holograms; in this device $p=-q$ and the far-field condition is

t The values of parameters are such that fine details are recorded. The source has effective angular diameter $d / p=1.6 \times 10^{-5}$, the pinhole diameter $\delta \approx \frac{1}{2} \mathrm{~mm}$, $b \approx 14 \mathrm{~mm}$ is the distance from the pinhole to far edge of the triangular array, the mean wavelength $\lambda \approx 5790 \AA$ and $\Delta \lambda \approx 100 \AA$. automatically fulfilled. In the $\mathrm{X}$-ray case, however, lenses are not available and the far-field condition severely restricts object size. If, for example, $\lambda=\frac{3}{2} \AA$ and $p=q=1 \mathrm{~m}$, then the farfield condition specifies an object of diameter $A=\frac{1}{2} b \leqslant 5 \mu$. However this condition can be relaxed by recording the $\mathrm{X}$-ray hologram in the Fresnel diffraction region of the object. We require that the diffracted waves from the object and pinhole overlap sufficiently so that phase information is recorded. The overlap condition (which replaces the far-field condition) is

$$
2 / \lambda|1 / p+1 / q| b \delta \leqslant 1 \text {. }
$$

Therefore a somewhat larger object (or smaller $p$ and $q$ ) can be used, and if $\lambda=\frac{3}{2} \AA, p=q=1 \mathrm{~m}$, and $\delta=1 / 10 \mu$, then $A \leqslant 190 \mu$. The hologram made under these conditions is reconstructed by optical Fourier transformation. The amplitudes of the reconstruction images differ from $R(u)$ and $R^{*}(-\boldsymbol{c})$ by an unobservable quadratic phase factor.

\section{References}

1. D.Gabor, Proc.Roy.Soc. (London) A197 (1949) 454 .

2. E.N. Leith and J. Upatnieks, J.Opt.Soc. Am. 52 (1962) 1123

3. J.T.Winthrop and C.R.Worthington, Physics Letters 15 (1965) 124

4. J.T.Winthrop and C.R. Worthington, J. Opt.Soc. Am. , to be published.

5. E.N. Leith and J. Upatnieks, J. Opt. Soc. Am. 54 (1964) 1295.

6. A.Vander Lugt, I.E.E.E., I.T.10 (1964) 138.

\title{
FREQUENCY SHIFTS IN THE RAMAN LINES DUE TO THE HIGH-FREQUENCY STARK EFFECT
}

\author{
M.K.DHEER and T.S.JASEJA \\ Indian Institute of Technology, Kanpur, India
}

Received 3 May 1966

\begin{abstract}
Experimental observations are reported on the shifts of the first order Raman lines arising from the Stark effect produced by a large field. The effect was pointed out by Javan.
\end{abstract}

The stimulated Raman effects produced in a few liquids were studied in some detail. Out of these liquids, cyclohexane showed an interesting
Raman effect [1]. When the Raman cell was well aligned parallel to the front maser-mirror of the ruby laser, another molecular vibration $[1,2]$ 